TRANSACTIONS OF THE

AMERICAN MATHEMATICAL SOCIETY

Volume 361, Number 12, December 2009, Pages 6191-6203

S 0002-9947(09)04642-X

Article electronically published on July 22, 2009

\title{
IMPROVED HARDY AND RELLICH INEQUALITIES ON RIEMANNIAN MANIFOLDS
}

\author{
ISMAIL KOMBE AND MURAD ÖZAYDIN
}

\begin{abstract}
In this paper we establish improved Hardy and Rellich type inequalities on a Riemannian manifold $M$. Furthermore, we also obtain sharp constants for improved Hardy and Rellich type inequalities on the hyperbolic space $\mathbb{H}^{n}$.
\end{abstract}

\section{INTRODUCTION}

The classical Hardy inequality states that for $n \geq 3$

$$
\int_{\mathbb{R}^{n}}|\nabla \phi(x)|^{2} d x \geq\left(\frac{n-2}{2}\right)^{2} \int_{\mathbb{R}^{n}} \frac{|\phi(x)|^{2}}{|x|^{2}} d x,
$$

where $\phi \in C_{c}^{\infty}\left(\mathbb{R}^{n} \backslash\{0\}\right)$ and the constant $\left(\frac{n-2}{2}\right)^{2}$ is sharp. An extension of Hardy's inequality to the second-order derivative is the Rellich inequality

$$
\int_{\mathbb{R}^{n}}|\Delta \phi(x)|^{2} d x \geq \frac{n^{2}(n-4)^{2}}{16} \int_{\mathbb{R}^{n}} \frac{|\phi(x)|^{2}}{|x|^{4}} d x
$$

for all $\phi \in C_{c}^{\infty}\left(\mathbb{R}^{n} \backslash\{0\}\right)$ and $n \neq 2$, where the constant $\frac{n^{2}(n-4)^{2}}{16}$ is sharp.

There has been a lot of research concerning Hardy and Rellich inequalities on the Euclidean space $\mathbb{R}^{n}$ and, in particular, sharp inequalities as well as their improved versions (in the sense that nonnegative terms are added in the right hand side of (1.1) and (1.2)), which have attracted a lot of attention because of their application to singular problems, e.g. [2, [21, [5], 13, [24, 6], 10] and the references therein. In recent years, some attention has been paid to Hardy and Rellich inequalities in Sub-Riemmannian spaces, e.g. 16 and the references therein. There is less literature for a general Riemannian manifold. In an interesting paper, Carron [7] studied weighted $L^{2}$-Hardy inequalities under some geometric assumptions on the weight function $\rho$ and obtained, among other results, the following inequality:

$$
\int_{M} \rho^{\alpha}|\nabla \phi|^{2} d x \geq\left(\frac{C+\alpha-1}{2}\right)^{2} \int_{M} \rho^{\alpha} \frac{\phi^{2}}{\rho^{2}} d x
$$

where $\alpha \in \mathbb{R}, C+\alpha-1>0, \phi \in C_{c}^{\infty}\left(M-\rho^{-1}\{0\}\right)$ and the weight function $\rho$ satisfies $|\nabla \rho|=1$ and $\Delta \rho \geq \frac{C}{\rho}$ in the sense of distribution.

Received by the editors March 13, 2007.

2000 Mathematics Subject Classification. Primary 26D10; Secondary 53C21.

Key words and phrases. Hardy inequality, Rellich inequality, sharp constants.

(C)2009 American Mathematical Society 
Under these geometric assumptions, our first goal is to obtain an $L^{p}$-version of the weighted Hardy inequality (1.3). This result plays an important role in the proof of the improved Hardy inequality (see Theorem 2.3). We also prove improved Rellich and uncertainty principle type inequalities. We should mention that Davies and Hinz [8] studied $L^{p}$-Rellich type inequalities, as well as their higher order versions. In [14, Grillo obtained Hardy, Rellich and Sobolev inequalities in the context of homogeneous spaces. Similarly, in [19, 20], 9], geometric properties such as volume growth and isoperimetric profile are related to the existence of Hardy type inequalities. A detailed treatment of improved Hardy inequalities with best constants, involving various kinds of distance functions in the Euclidean space $\mathbb{R}^{n}$, can be found in [4. Recently, Barbatis [3] obtained, under a geometric assumption, an improved higher-order Rellich inequality of the form

$$
\int_{\Omega} \frac{\left|\Delta^{m / 2} u\right|^{p}}{d^{\gamma}} d x \geq A(m, \gamma) \int_{\Omega} \frac{|u|^{p}}{d^{\gamma+m p}} d x+B(m, \gamma) \int_{\Omega} V_{i}|u|^{p} d x
$$

where $u \in C_{c}^{\infty}(\Omega \backslash K), d(x)=\operatorname{dist}(x, K), \gamma \in \mathbb{N}$ and $V_{i}(x)$ involves some suitable iterated logarithmic functions. Our improved Rellich inequality, Theorem 2.5 below, has a different type of a remainder term involving gradient $\nabla u$.

Let us recall that a complete Riemannian manifold $M$ is said to be nonparabolic if there exists a symmetric positive Green's function $G(x, y)$ for the Laplacian $\Delta$ on $L^{2}$ functions. Recently, Li and Wang [17] proved that, among other results, existence of a weighted Hardy type inequality is equivalent to nonparabolicity. Furthermore, they obtained the following $L^{2}$-Hardy inequality:

$$
\int_{M}|\nabla \phi|^{2} d x \geq \int_{M} \frac{|\nabla G(p, x)|^{2}}{4 G^{2}(p, x)} \phi^{2} d x
$$

where $\phi \in C_{c}^{\infty}(M)$ and $G(p, x)$ is the minimal positive Green's function defined on $M$ with a pole at the point $p \in M$.

The second goal of this paper is to find sharp versions of improved Hardy and Rellich inequalities in the specific case of the hyperbolic spaces $\mathbb{H}^{n}$. Both Hardy and Rellich inequalities in hyperbolic spaces as well as the determination of sharp constants is new.

\section{Improved HaRdy and Rellich inequalities ON RIEMANNIAN MANIFOLDS}

In the various integral inequalities below (Section 2 and Section 3), we allow the values of the integrals on the left hand sides to be $+\infty$. The following theorem is the first result of this section.

Theorem 2.1. Let $M$ be a complete noncompact Riemannian manifold of dimension $n>1$. Let $\rho$ be a nonnegative function on $M$ such that $|\nabla \rho|=1$ and $\Delta \rho \geq \frac{C}{\rho}$ in the sense of distribution where $C>0$. Then the following inequality holds:

$$
\int_{M} \rho^{\alpha}|\nabla \phi|^{p} d x \geq\left(\frac{C+1+\alpha-p}{p}\right)^{p} \int_{M} \rho^{\alpha} \frac{|\phi|^{p}}{\rho^{p}} d x
$$

for all compactly supported smooth functions $\phi \in C_{c}^{\infty}\left(M \backslash \rho^{-1}\{0\}\right), 1 \leq p<\infty$, and $C+1+\alpha-p>0$.

Proof. Let $\phi=\rho^{\gamma} \psi$, where $\psi \in C_{c}^{\infty}(M)$ and $\gamma<0$. We have

$$
\left|\nabla\left(\rho^{\gamma} \psi\right)\right|=\left|\gamma \rho^{\gamma-1} \psi \nabla \rho+\rho^{\gamma} \nabla \psi\right| .
$$


We now use the following inequality which is valid for any $a, b \in \mathbb{R}^{n}$ and $p>2$ :

$$
|a+b|^{p}-|a|^{p} \geq c(p)|b|^{p}+p|a|^{p-2} a \cdot b,
$$

where $c(p)>0$. This yields

$$
\rho^{\alpha}|\nabla \phi|^{p} \geq|\gamma|^{p} \rho^{\gamma p-p+\alpha}|\psi|^{p}+p|\gamma|^{p-2} \gamma \rho^{\alpha+\gamma p+1-p}|\psi|^{p-2} \psi \nabla \rho \cdot \nabla \phi .
$$

Then integration by parts gives

$$
\begin{gathered}
\int_{M} \rho^{\alpha}|\nabla \phi|^{p} d x \geq|\gamma|^{p} \int_{M} \rho^{\gamma p-p+\alpha}|\psi|^{p} d x-\frac{|\gamma|^{p-2} \gamma}{\alpha+\gamma p-p+2} \int_{M} \Delta\left(\rho^{\alpha+\gamma p-p+2}\right)|\psi|^{p} d x \\
\geq(1-p)|\gamma|^{p} \int_{M} \rho^{\gamma p-p+\alpha}|\psi|^{p} d x-\gamma|\gamma|^{p-2}(\alpha+C+1-p) \int_{M} \rho^{\gamma p-p+\alpha}|\psi|^{p} d x
\end{gathered}
$$

We now choose $\gamma=\frac{p-\alpha-C-1}{p}$ to get the desired inequality

$$
\int_{M} \rho^{\alpha}|\nabla \phi|^{p} d x \geq\left(\frac{C+1+\alpha-p}{p}\right)^{p} \int_{M} \rho^{\alpha} \frac{|\phi|^{p}}{\rho^{p}} d x .
$$

Theorem 2.1 also holds for $1<p<2$, and in this case we use the following inequality:

$$
|a+b|^{p}-|a|^{p} \geq c(p) \frac{|b|^{2}}{(|a|+|b|)^{2-p}}+p|a|^{p-2} a \cdot b,
$$

where $c(p)>0$ (see [18]).

We now prove the following theorem which also contains Hardy's inequality.

Theorem 2.2. Let $M$ be a complete noncompact Riemannian manifold of dimension $n>1$. Let $\rho$ be a nonnegative function on $M$ such that $|\nabla \rho|=1$ and $\Delta \rho \geq \frac{C}{\rho}$, where $C>q-1$. Then the following inequality holds:

$$
\left(\int_{M}|\phi|^{p} d x\right)^{\frac{p-q}{p}}\left(\int_{M}|\nabla \phi|^{p}\right)^{\frac{q}{p}} d x \geq\left(\frac{C+1-q}{p}\right)^{q} \int_{M} \frac{|\phi|^{p}}{\rho^{q}} d x
$$

for all compactly supported smooth functions $\phi \in C_{c}^{\infty}\left(M \backslash \rho^{-1}\{0\}\right)$, where $1 \leq p<$ $\infty$ and $0 \leq q \leq p$.

Proof. The argument is a simple application of the divergence theorem:

Let $Q(x)=\frac{\nabla \rho}{\rho^{q-1}}$; then we have $\nabla \cdot Q \geq \frac{(C+1-q)}{\rho^{q}}$. It is clear that

$$
\nabla \cdot\left(|\phi|^{p} Q(x)\right)=p|\phi|^{p-1} \nabla|\phi| \cdot Q(x)+|\phi|^{p} \nabla \cdot Q(x) .
$$

Integrating the above formula, we get

$$
(C+1-q) \int_{M} \frac{|\phi|^{p}}{\rho^{q}} d x \leq p \int_{M} \frac{|\phi|^{p-1}}{\rho^{q-1}}|\nabla \phi| d x .
$$

Applying Hölder's inequality, we obtain the desired inequality:

$$
\left(\int_{M}|\phi|^{p} d x\right)^{\frac{p-q}{p}}\left(\int_{M}|\nabla \phi|^{p} d x\right)^{\frac{q}{p}} \geq\left(\frac{C+1-q}{p}\right)^{q} \int_{M} \frac{|\phi|^{p}}{\rho^{q}} d x .
$$

We now prove the following improved Hardy inequality which is inspired by recent work of Abdellaoui, Colorado and Peral [1]. 
Theorem 2.3. Let $\Omega$ be a bounded domain with smooth boundary $\partial \Omega$ in a complete noncompact Riemmannian manifold of dimension $n>1$. Let $\rho$ be a nonnegative function on $\Omega$ such that $|\nabla \rho|=1$ and $\Delta \rho \geq \frac{C}{\rho}$ in the sense of distribution, where $C>0,1<q<2, \alpha \in \mathbb{R}, C+\alpha-1>0$. Then there exists a positive constant $C_{1}=C_{1}(n, q, \Omega)$ such that the following inequality holds:

$$
\int_{\Omega} \rho^{\alpha}|\nabla \phi|^{2} d x \geq\left(\frac{C+\alpha-1}{2}\right)^{2} \int_{\Omega} \rho^{\alpha} \frac{\phi^{2}}{\rho^{2}} d x+C_{1}\left(\int_{\Omega}|\nabla \phi|^{q} \rho^{q \alpha / 2} d x\right)^{2 / q}
$$

for all compactly supported smooth functions $\phi \in C_{c}^{\infty}(\Omega)$.

Proof. Let $\phi \in C_{c}^{\infty}(\Omega)$ and $\psi=\rho^{\beta}$ where $\beta<0$. Then a straightforward computation shows that

$$
|\nabla \phi|^{2}-\nabla\left(\frac{\phi^{2}}{\psi}\right) \cdot \nabla \psi=\left|\nabla \phi-\frac{\phi}{\psi} \nabla \psi\right|^{2} .
$$

Therefore

$$
\begin{aligned}
\int_{\Omega}\left(|\nabla \phi|^{2}-\nabla\left(\frac{\phi^{2}}{\psi}\right) \cdot \nabla \psi\right) \rho^{\alpha} d x & =\int_{\Omega}\left|\nabla \phi-\frac{\phi}{\psi} \nabla \psi\right|^{2} \rho^{\alpha} d x \\
& \geq C_{1}\left(\int_{\Omega}\left|\nabla \phi-\frac{\phi}{\psi} \nabla \psi\right|^{q} \rho^{q \alpha / 2} d x\right)^{2 / q},
\end{aligned}
$$

where we used Jensen's inequality in the last step. It is clear that

$$
\begin{aligned}
\int_{\Omega}\left(|\nabla \phi|^{2}-\nabla\left(\frac{\phi^{2}}{\psi}\right) \cdot \nabla \psi\right) \rho^{\alpha} d x & =\int_{\Omega} \rho^{\alpha}|\nabla \phi|^{2} d x+\frac{\beta}{\alpha+\beta} \int_{\Omega}\left(\frac{\Delta\left(\rho^{\alpha+\beta}\right)}{\rho^{\beta}}\right) \phi^{2} d x \\
& \leq \int_{\Omega} \rho^{\alpha}|\nabla \phi|^{2} d x+\beta(\alpha+\beta+C-1) \int_{\Omega} \rho^{\alpha-2} \phi^{2} d x
\end{aligned}
$$

Therefore we have

$$
\begin{aligned}
\int_{\Omega} \rho^{\alpha}|\nabla \phi|^{2} d x & \geq-\beta^{2}-\beta(\alpha+C-1) \int_{\Omega} \rho^{\alpha} \frac{\phi^{2}}{\rho^{2}} d x \\
& +C_{1}\left(\int_{\Omega}\left|\nabla \phi-\frac{\phi}{\psi} \nabla \psi\right|^{q} \rho^{q \alpha / 2} d x\right)^{2 / q} .
\end{aligned}
$$

Now we can use the following elementary inequality: Let $1<q<2$ and $w_{1}, w_{2} \in \mathbb{R}^{n}$; then the following holds:

$$
c(q)\left|w_{2}\right|^{q} \geq\left|w_{1}+w_{2}\right|^{q}-\left|w_{1}\right|^{q}-q\left|w_{1}\right|^{q-2}\left\langle w_{1}, w_{2}\right\rangle .
$$

Now, integrating and using successively the inequality (2.5), Young's inequality and weighted $L^{p}$-Hardy inequality $(2.1)$, we get

$$
\int_{\Omega}\left|\nabla \phi-\frac{\phi}{\psi} \nabla \psi\right|^{q} \rho^{q \alpha / 2} d x \geq C_{1} \int_{\Omega}|\nabla \phi|^{q} \rho^{q \alpha / 2} d x .
$$

Substituting (2.6) into (2.4) we get

$$
\int_{\Omega} \rho^{\alpha}|\nabla \phi|^{2} d x \geq-\beta^{2}-\beta(\alpha+C-1) \int_{\Omega} \rho^{\alpha} \frac{\phi^{2}}{\rho^{2}} d x+C_{1}\left(\int_{\Omega}|\nabla \phi|^{q} \rho^{q \alpha / 2} d x\right)^{2 / q} .
$$

Now choosing $\beta=\frac{1-\alpha-C}{2}$, we obtain the desired inequality:

$$
\int_{\Omega} \rho^{\alpha}|\nabla \phi|^{2} d x \geq\left(\frac{C+\alpha-1}{2}\right)^{2} \int_{\Omega} \rho^{\alpha} \frac{\phi^{2}}{\rho^{2}} d x+C_{1}\left(\int_{\Omega}|\nabla \phi|^{q} \rho^{q \alpha / 2} d x\right)^{2 / q} .
$$


We now turn our attention to the Rellich type inequalities. We first prove the following plain weighted Rellich inequality on $M$.

Theorem 2.4 (Weighted Rellich inequality). Let $M$ be a complete noncompact Riemannian manifold of dimension $n>1$. Let $\rho$ be a nonnegative function on $M$ such that $|\nabla \rho|=1$ and $\Delta \rho \geq \frac{C}{\rho}$ in the sense of distribution where $C>0$. Then the following inequality holds:

$$
\int_{M} \rho^{\alpha}|\Delta \phi|^{2} d x \geq \frac{(C+\alpha-3)^{2}(C-\alpha+1)^{2}}{16} \int_{M} \rho^{\alpha} \frac{\phi^{2}}{\rho^{4}} d x
$$

for all compactly supported smooth functions $\phi \in C_{c}^{\infty}\left(M \backslash \rho^{-1}\{0\}\right), \alpha<2$ and $C+\alpha-3>0$.

Proof. A straightforward computation shows that

$$
\Delta \rho^{\alpha-2} \leq(\alpha-2)(C+\alpha-3) \rho^{\alpha-4} .
$$

Multiplying both sides of (2.8) by $\phi^{2}$ and integrating over $M$, we obtain

$$
\begin{aligned}
(C+\alpha-3)(\alpha-2) \int_{M} \rho^{\alpha-4} \phi^{2} d x & \geq \int_{M} \rho^{\alpha-2} \Delta\left(\phi^{2}\right) d x \\
& =\int_{M} \rho^{\alpha-2}\left(2|\nabla \phi|^{2}+2 \phi \Delta \phi\right) d x
\end{aligned}
$$

Therefore

(2.10) $-2 \int_{M}(\phi \Delta \phi) \rho^{\alpha-2} d x \geq 2 \int_{M} \rho^{\alpha-2}|\nabla \phi|^{2} d x-(\alpha-2)(C+\alpha-3) \int_{M} \rho^{\alpha-4} \phi^{2} d x$.

After applying the weighted Hardy (1.3) and Cauchy-Schwarz inequalities, we obtain the following weighted Rellich inequality:

$$
\int_{M} \rho^{\alpha}|\Delta \phi|^{2} d x \geq \frac{(C+\alpha-3)^{2}(C-\alpha+1)^{2}}{16} \int_{M} \rho^{\alpha} \frac{\phi^{2}}{\rho^{4}} d x
$$

We now obtain an improved version of (2.7) for a bounded domain in $M$.

Theorem 2.5 (Improved weighted Rellich inequality). Let $\Omega$ be a bounded domain with smooth boundary $\partial \Omega$ in a complete noncompact Riemmannian manifold of dimension $n>1$. Let $\rho$ be a nonnegative function on $\Omega$ such that $|\nabla \rho|=1$ and $\Delta \rho \geq \frac{C}{\rho}$ in the sense of distribution, where $C>0,1<q<2, \alpha<2$ and $C+\alpha-3>0$. Then there exists a positive constant $C_{1}=C_{1}(n, q, \Omega)$ such that the following inequality holds:

$$
\begin{aligned}
\int_{\Omega} \rho^{\alpha}|\Delta \phi|^{2} d x & \geq \frac{(C+\alpha-3)^{2}(C-\alpha+1)^{2}}{16} \int_{\Omega} \rho^{\alpha} \frac{\phi^{2}}{\rho^{4}} d x \\
& +\frac{(C+\alpha-3)(C-\alpha+1)}{2} C_{1}\left(\int_{\Omega}|\nabla \phi|^{q} \rho^{\frac{q(\alpha-2)}{2}} d x\right)^{2 / q}
\end{aligned}
$$

for all compactly supported smooth functions $\phi \in C_{c}^{\infty}(\Omega)$.

Proof. The proof of this theorem is quite similar to the proof of Theorem 2.4; the only difference is that we use improved Hardy inequality (2.3) instead of (1.3). Using the same argument as above, we have the following from (2.10):

$(2.11)-2 \int_{\Omega}(\phi \Delta \phi) \rho^{\alpha-2} d x \geq 2 \int_{\Omega} \rho^{\alpha-2}|\nabla \phi|^{2} d x-(\alpha-2)(C+\alpha-3) \int_{\Omega} \rho^{\alpha-4} \phi^{2} d x$. 
Applying Young's inequality to the expression $-\int_{M}(\phi \Delta \phi) \rho^{\alpha-2}$ in (2.11) we obtain

$$
-\int_{M} \rho^{\alpha-2} \phi \Delta \phi d x \leq \epsilon \int_{M} \rho^{\alpha-4} \phi^{2} d x+\frac{1}{4 \epsilon} \int_{M} \rho^{\alpha}|\Delta \phi|^{2} d x
$$

where $\epsilon>0$. Substituting (2.12) into (2.11) and using the improved Hardy inequality (2.3), we get

$$
\begin{aligned}
\int_{\Omega} \rho^{\alpha}|\Delta \phi|^{2} d x & \geq\left(-4 \epsilon^{2}+\epsilon(C+\alpha-3)(C-\alpha+1)\right) \int_{\Omega} \rho^{\alpha-4} \phi^{2} d x \\
& +4 \epsilon C_{1}\left(\int_{\Omega}|\nabla \phi|^{q} \rho^{\frac{q(\alpha-2)}{2}} d x\right)^{2 / q} .
\end{aligned}
$$

Note that the quadratic function $-4 \epsilon^{2}+\epsilon(C+\alpha-3)(C-\alpha+1)$ attains the maximum for $\epsilon=\frac{(C+\alpha-3)(C-\alpha+1)}{8}$, and this maximum is equal to $\frac{(C+\alpha-3)^{2}(C-\alpha+1)^{2}}{16}$. Therefore we obtain the following improved weighted Rellich inequality:

$$
\begin{aligned}
\int_{\Omega} \rho^{\alpha}|\Delta \phi|^{2} d x & \geq \frac{(C+\alpha-3)^{2}(C-\alpha+1)^{2}}{16} \int_{\Omega} \rho^{\alpha} \frac{\phi^{2}}{\rho^{4}} d x \\
& +\frac{(C+\alpha-3)(C-\alpha+1)}{2} C_{1}\left(\int_{\Omega}|\nabla \phi|^{q} \rho^{\frac{q(\alpha-2)}{2}} d x\right)^{2 / q} .
\end{aligned}
$$

Uncertainty principle inequality. The classical uncertainty principle was developed in the context of quantum mechanics by Heisenberg [15. It says that the position and momentum of a particle cannot be determined exactly at the same time but can only be determined with an "uncertainty". The harmonic analysis version of the uncertainty principle states that a function on the real line and its Fourier transform cannot be simultaneously well localized. It has been widely studied in quantum mechanics and signal analysis. There are various forms of the uncertainty principle. For an overview we refer to Folland's and Sitaram's paper [11.

The uncertainty principle on the Euclidean space $\mathbb{R}^{n}$ can be stated in the following way:

$$
\left(\int_{\mathbb{R}^{n}}|x|^{2}|f(x)|^{2} d x\right)\left(\int_{\mathbb{R}^{n}}|\nabla f(x)|^{2} d x\right) \geq \frac{n^{2}}{4}\left(\int_{\mathbb{R}^{n}}|f(x)|^{2} d x\right)^{2}
$$

for all $f \in L^{2}\left(\mathbb{R}^{n}\right)$.

Using Hardy type inequalities, we obtain the following uncertainty principle type inequalities on a Riemannian manifold $M$.

Corollary 2.1 ( $L^{p}$-uncertainty principle inequality). Let $M$ be a complete noncompact Riemannian manifold of dimension $n>1$. Let $\rho$ be a nonnegative function on $M$ such that $|\nabla \rho|=1$ and $\Delta \rho \geq \frac{C}{\rho}$ in the sense of distribution where $C>0$. Then the following inequality holds:

$$
\left(\int_{M} \rho^{q} \phi^{q} d x\right)^{1 / q}\left(\int_{M}|\nabla \phi|^{p} d x\right)^{1 / p} \geq \frac{C+1-p}{p} \int_{M} \phi^{2} d x
$$

for all compactly supported smooth functions $\phi \in C_{c}^{\infty}\left(M \backslash \rho^{-1}\{0\}\right), 1<p<\infty$, $\frac{1}{p}+\frac{1}{q}=1$ and $C+1-p>0$.

Corollary 2.2 (Improved $L^{2}$-uncertainty principle inequality). Let $\Omega$ be a bounded domain with smooth boundary $\partial \Omega$ in a complete noncompact Riemmannian manifold of dimension $n>1$. Let $\rho$ be a nonnegative function on $\Omega$ such that $|\nabla \rho|=1$ 
and $\Delta \rho \geq \frac{C}{\rho}$ in the sense of distribution where $C>0$. Then the following inequality holds:

$$
\left(\int_{\Omega} \rho^{\alpha} \phi^{2} d x\right)\left(\int_{\Omega} \rho^{\alpha}|\nabla \phi|^{2} d x-C_{1}\left(\int_{\Omega}|\nabla \phi|^{2} \rho^{\frac{\alpha q}{2}}\right)^{2 / q}\right) \geq\left(\frac{C+\alpha-1}{2}\right)^{2}\left(\int_{\Omega} \phi^{2} d x\right)^{2}
$$

for all compactly supported smooth functions $\phi \in C_{c}^{\infty}(\Omega), 1<q<2, C+\alpha-1>0$ and $C_{1}>0$.

\section{Sharp improved Hardy and Rellich inequalities ON HYPERBOLIC SPACE $\mathbb{H}^{n}$}

In this section we obtain sharp constants for improved Hardy and Rellich type inequalities on the hyperbolic space $\mathbb{H}^{n}$. We will be using the Poincaré conformal disc model for the hyperbolic space $\mathbb{H}^{n}$. So the underlying space is

$$
\mathbb{B}^{n}=\left\{x=\left(x_{1}, \cdots, x_{n}\right) \in \mathbb{R}^{n}|| x \mid<1\right\}
$$

in $\mathbb{R}^{n}$ equipped with the Riemannian metric obtained by scaling the Euclidean metric with a factor of $p:=\frac{2}{1-|x|^{2}}$. Hence $\left\{p d x_{i}\right\}_{i=1}^{n}$ give an orthonormal basis of the cotangent space at $x=\left(x_{1}, \cdots, x_{n}\right)$ in $\mathbb{B}^{n}$. The corresponding dual basis is $\left\{\frac{1}{p} \frac{\partial}{\partial x_{i}}\right\}_{i=1}^{n}$; thus the hyperbolic gradient is

$$
\nabla_{\mathbb{H}^{n}} u=\frac{\nabla u}{p},
$$

where $u \in C^{1}\left(\mathbb{B}^{n}\right)$ and $\nabla u$ is the usual gradient. $\mathbb{H}^{n}$ is a contractible complete Riemannian manifold with all sectional curvatures equal -1 . Geodesic lines passing through the origin are the diameters of $\mathbb{B}^{n}$ along with open arcs of circles in $\mathbb{B}^{n}$ perpendicular to the boundary at $\infty, \partial \mathbb{B}^{n}=S^{n-1}=\left\{x \in \mathbb{R}^{n}:|x|=1\right\}$. It follows that the distance from $x \in \mathbb{B}^{n}$ to the origin is

$$
d=d_{\mathbb{H}}(0, x)=\log \left(\frac{1+|x|}{1-|x|}\right) .
$$

The hyperbolic volume element is

$$
d V=p^{n}(x) d x
$$

(where $d x$ is the usual Euclidean volume element), and the Laplace-Beltrami operator is given by

$$
\Delta_{\mathbb{H}^{n}} u=p^{-n} \operatorname{div}\left(p^{n-2} \nabla u\right),
$$

where $\nabla$ and div denote the Euclidean gradient and divergence in $\mathbb{R}^{n}$, respectively.

Note that we have the following two relations for the distance function $d=$ $\log \left(\frac{1+|x|}{1-|x|}\right)$ :

$$
\begin{aligned}
\left|\nabla_{\mathbb{H}^{n}} d\right| & =1, \\
\Delta_{\mathbb{H}^{n}} d & \geq \frac{n-1}{d}, \quad x \neq 0 .
\end{aligned}
$$

Let us remark that the Poincaré inequality with the Muckenhoupt weight plays an important role in the following theorem. We recall that a weight $w(x)$ satisfies the Muckenhoupt $A_{p}$ condition for $1<p<\infty$ if there is a constant $C$ such that

$$
\left(\frac{1}{|B|} \int_{B} w(x) d x\right)^{1 / p}\left(\frac{1}{|B|} \int_{B} w(x)^{-p^{\prime} / p} d x\right)^{\frac{1}{p^{\prime}}} \leq C
$$


for all balls $B$. If $w(x) \in A_{p}$, then we have $w(x)^{-p^{\prime} / p} \in A_{p^{\prime}}$, where $p^{\prime}$ is the dual exponent to $p$ given by $\frac{1}{p}+\frac{1}{p^{\prime}}=1$.

Before we state our first theorem, let us mention a well known result of Brezis and Vázquez [5] in this direction. They proved that for a bounded domain $\Omega \subset \mathbb{R}^{n}$

$$
\int_{\Omega}|\nabla \phi(x)|^{2} d x \geq\left(\frac{n-2}{2}\right)^{2} \int_{\Omega} \frac{|\phi(x)|^{2}}{|x|^{2}} d x+\mu\left(\frac{\omega_{n}}{|\Omega|}\right)^{2 / n} \int_{\Omega} \phi^{2} d x
$$

where $\omega_{n}$ and $|\Omega|$ denote the $n$ dimensional Lebesgue measure of the unit ball $B \subset \mathbb{R}^{n}$ and the domain $\Omega$ respectively. Here $\mu$ is the first eigenvalue of the Laplace operator in the two dimensional unit disk, and it is optimal when $\Omega$ is a ball centered at the origin. We now prove a similar (weighted) improved Hardy inequality on hyperbolic space $\mathbb{H}^{n}$.

Theorem 3.1. Let $\alpha \in \mathbb{R}$ and $\phi \in C_{c}^{\infty}\left(\mathbb{H}^{n} \backslash\{0\}\right)$. Then we have

$$
\int_{\mathbb{B}^{n}} d^{\alpha}|\nabla \phi|^{2} p^{n-2} d x \geq\left(\frac{n+\alpha-2}{2}\right)^{2} \int_{\mathbb{B}^{n}} d^{\alpha} \frac{\phi^{2}}{d^{2}} p^{n} d x+c 2^{n-2} \int_{\mathbb{B}^{n}} d^{\alpha} \phi^{2} d x,
$$

where $d=\log \left(\frac{1+|x|}{1-|x|}\right)$ is the distance from $x \in \mathbb{B}^{n}$ to the origin and $c>0$. Moreover, the constant $\left(\frac{n+\alpha-2}{2}\right)^{2}$ is sharp provided $n+\alpha-2>0$.

Proof. Let $\phi=d^{\beta} \psi$, where $\beta \in \mathbb{R} \backslash\{0\}$ and $\psi \in C_{c}^{\infty}\left(\mathbb{H}^{n} \backslash\{0\}\right)$. A direct calculation shows that

$$
\begin{aligned}
d^{\alpha}|\nabla \phi|^{2} p^{n-2} & =\beta^{2} d^{\alpha+2 \beta-2}|\nabla d|^{2} \psi^{2} p^{n-2} \\
& +2 \beta d^{\alpha+2 \beta-1} \psi p^{n-2} \nabla d \cdot \nabla \psi+d^{\alpha+2 \beta}|\nabla \psi|^{2} p^{n-2} .
\end{aligned}
$$

It is easy to see that

$$
|\nabla d|^{2}=p^{2}
$$

and integrating $(3.2)$ over $\mathbb{B}^{n}$, we get

$$
\begin{aligned}
\int_{\mathbb{B}^{n}} d^{\alpha}|\nabla \phi|^{2} p^{n-2} d x & =\int_{\mathbb{B}^{n}} \beta^{2} d^{\alpha+2 \beta-2} \psi^{2} p^{n} d x+\int_{\mathbb{B}^{n}} 2 \beta d^{\alpha+2 \beta-1} \psi p^{n-2} \nabla d \cdot \nabla \psi d x \\
& +\int_{\mathbb{B}^{n}} d^{\alpha+2 \beta}|\nabla \psi|^{2} p^{n-2} d x
\end{aligned}
$$

Applying integration by parts to the middle integral on the right hand side of (3.3), we obtain

$$
\begin{aligned}
\int_{\mathbb{B}^{n}} d^{\alpha}|\nabla \phi|^{2} p^{n-2} d x & =\int_{\mathbb{B}^{n}} \beta^{2} d^{\alpha+2 \beta-2} \psi^{2} p^{n} d x-\frac{\beta}{\alpha+2 \beta} \int_{\mathbb{B}^{n}} \operatorname{div}\left(p^{n-2} \nabla\left(d^{2 \beta+\alpha}\right)\right) d x \\
& +\int_{\mathbb{B}^{n}} d^{\alpha+2 \beta}|\nabla \psi|^{2} p^{n-2} d x
\end{aligned}
$$

One can show that

$$
\begin{aligned}
& -\frac{\beta}{\alpha+2 \beta} \int_{\mathbb{B}^{n}} \operatorname{div}\left(p^{n-2} \nabla\left(d^{2 \beta+\alpha}\right)\right) d x \\
= & -\beta(2 \beta+\alpha-1) \int_{\mathbb{B}^{n}} d^{2 \beta+\alpha-2} p^{n} \psi^{2} d x-\beta \int_{\mathbb{B}^{n}} d^{2 \beta+\alpha-1} p^{n-2} \psi^{2}(\Delta d) d x \\
& -\beta(n-2) \int_{\mathbb{B}^{n}} d^{2 \beta+\alpha-1} p^{n-3}(\nabla d \cdot \nabla p) d x .
\end{aligned}
$$


Also,

$$
\Delta d=p^{2} r+\frac{n-1}{r} p
$$

and

$$
\nabla d \cdot \nabla p=p^{3} r
$$

where $r=|x|$. Substituting the above,

$$
\begin{array}{r}
-\frac{\beta}{\alpha+2 \beta} \int_{\mathbb{B}^{n}} \operatorname{div}\left(p^{n-2} \nabla\left(d^{2 \beta+\alpha}\right)\right) d x=-\beta(2 \beta+\alpha-1) \int_{\mathbb{B}^{n}} d^{2 \beta+\alpha-2} p^{n} \psi^{2} d x \\
-(2 \beta+\alpha) \int_{\mathbb{B}^{n}} d^{2 \beta+\alpha-1} p^{n}\left(\frac{(n-1)\left(p r^{2}+1\right)}{p r}\right) \psi^{2} d x .
\end{array}
$$

We can easily show that

$$
\frac{p r^{2}+1}{p r} \geq \frac{1}{d}
$$

If $2 \beta+\alpha<0$, then we have

$$
-\frac{\beta}{\alpha+2 \beta} \int_{\mathbb{B}^{n}} \operatorname{div}\left(p^{n-2} \nabla\left(d^{2 \beta+\alpha}\right)\right) d x \geq-\beta(2 \beta+\alpha+n-2) \int_{\mathbb{B}^{n}} d^{2 \beta+\alpha-2} p^{n} \psi^{2} d x .
$$

Now we substitute (3.6) into (3.4), and we get

$$
\begin{aligned}
\int_{\mathbb{B}^{n}} d^{\alpha}|\nabla \phi|^{2} p^{n-2} d x \geq & \left(-\beta^{2}-\beta(\alpha+n-2)\right) \int_{\mathbb{B}^{n}} d^{2 \beta+\alpha-2} \psi^{2} p^{n} d x \\
& +\int_{\mathbb{B}^{n}} d^{\alpha+2 \beta}|\nabla \psi|^{2} p^{n-2} d x
\end{aligned}
$$

Note that the function $\beta \longrightarrow-\beta^{2}-\beta(\alpha+n-2)$ attains the maximum for $\beta=\frac{2-\alpha-n}{2}$, and this maximum is equal to $\left(\frac{n+\alpha-2}{2}\right)^{2}$. Therefore we have the following inequality:

$$
\begin{aligned}
\int_{\mathbb{B}^{n}} d^{\alpha}|\nabla \phi|^{2} p^{n-2} d x & \geq\left(\frac{n+\alpha-2}{2}\right)^{2} \int_{\mathbb{B}^{n}} d^{\alpha} \frac{\phi^{2}}{d^{2}} p^{n} d x+\int_{\mathbb{B}^{n}} d^{2-n}|\nabla \psi|^{2} p^{n-2} d x \\
& \geq\left(\frac{n+\alpha-2}{2}\right)^{2} \int_{\mathbb{B}^{n}} d^{\alpha} \frac{\phi^{2}}{d^{2}} p^{n} d x+\int_{\mathbb{B}^{n}} r^{2-n}|\nabla \psi|^{2} d x .
\end{aligned}
$$

Notice that the weight function $r^{2-n}$ is in the Muckenhoupt $A_{2}$ class and that we have the weighted Poincaré inequality [12. Therefore

$$
\int_{\mathbb{B}^{n}} d^{\alpha}|\nabla \phi|^{2} d x \geq\left(\frac{n+\alpha-2}{2}\right)^{2} \int_{\mathbb{B}^{n}} d^{\alpha} \frac{\phi^{2}}{d^{2}} p^{n} d x+c \int_{\mathbb{B}^{n}} r^{2-n} \psi^{2} d x,
$$

where $c>0$. Since $2 r \leq d \leq p r$, we obtain the following improved Hardy inequality:

$$
\int_{\mathbb{B}^{n}} d^{\alpha}|\nabla \phi|^{2} p^{n-2} d x \geq\left(\frac{n+\alpha-2}{2}\right)^{2} \int_{\mathbb{B}^{n}} d^{\alpha} \frac{\phi^{2}}{d^{2}} p^{n} d x+c 2^{n-2} \int_{\mathbb{B}^{n}} d^{\alpha} \phi^{2} d x
$$


It only remains to show that the constant $\left(\frac{n+\alpha-2}{2}\right)^{2}$ is the best constant for the Hardy inequality (3.8); that is,

$$
\left(\frac{n+\alpha-2}{2}\right)^{2}=\inf \left\{\frac{\int_{\mathbb{B}^{n}} d^{\alpha}|\nabla \phi|^{2} p^{n-2} d x}{\int_{\mathbb{B}^{n}} d^{\alpha-2} \phi^{2} p^{n} d x}, \phi \in C_{c}^{1}\left(\mathbb{B}^{n}\right), \phi \neq 0\right\} .
$$

Let $\phi_{\epsilon}(d)$ be the family of functions defined by

$$
\phi_{\epsilon}(d)= \begin{cases}1 & \text { if } \quad d \in[0,1], \\ d^{-\left(\frac{n+\alpha-2}{2}+\epsilon\right)} & \text { if } \quad d>1,\end{cases}
$$

where $\epsilon>0$ and $d=\log \left(\frac{1+|x|}{1-|x|}\right)$. It follows that

$$
\int_{\mathbb{B}^{n}} d^{\alpha}\left|\nabla \phi_{\epsilon}\right|^{2} p^{n-2} d x=\left(\frac{n+\alpha-2}{2}+\epsilon\right)^{2} \int_{\mathbb{B}^{n}} d^{-n-2 \epsilon} p^{n} d x .
$$

In the sequel $B_{1}=\{x: d \leq 1\}$, the $d$-ball centered at the origin in $\mathbb{B}^{n}$ with radius 1.

By direct computation we get

$$
\begin{aligned}
\int_{\mathbb{B}^{n}} d^{\alpha} \frac{\phi_{\epsilon}^{2}}{d^{2}} p^{n} d x & =\int_{B_{1}} d^{\alpha-2} p^{n} d x+\int_{\mathbb{B}^{n} \backslash B_{1}} d^{-n-2 \epsilon} p^{n} d x \\
& =\int_{B_{1}} d^{\alpha-2} p^{n} d x+\left(\frac{n+\alpha-2}{2}+\epsilon\right)^{-2} \int_{\mathbb{B}^{n}} d^{\alpha}\left|\nabla \phi_{\epsilon}\right|^{2} p^{n} d x .
\end{aligned}
$$

Since $n+\alpha-2>0$, the first integral on the right hand side of (3.10) is integrable and $\epsilon \longrightarrow 0$ yields the desired result.

Remark 3.2. Using Poincaré's inequality in the two dimensions above we obtain the following improved Hardy inequality which has an explicit constant for the remainder term:

$$
\int_{\mathbb{B}^{n}} d^{\alpha}|\nabla \phi|^{2} p^{n-2} d x \geq\left(\frac{n+\alpha-2}{2}\right)^{2} \int_{\mathbb{B}^{n}} d^{\alpha} \frac{\phi^{2}}{d^{2}} p^{n} d x+2^{n+\alpha-2} \mu \int_{\mathbb{B}^{n}} r^{\alpha} \phi^{2} d x,
$$

where $\mu$ is the first eigenvalue of the usual Laplace operator in two dimensional unit disk.

We now give a new improved version of uncertainty principle inequality on the hyperbolic space, which is an immediate consequence of the improved Hardy inequality (3.8) and the Cauchy-Schwarz inequality. Let us mention that a different version of the uncertainty principle inequality on hyperbolic space has been obtained by Sun $[23$.

Corollary 3.1 (Improved uncertainty principle inequality). Let $\phi \in C_{c}^{\infty}\left(\mathbb{B}^{n} \backslash\{0\}\right)$, $d=\log \left(\frac{1+|x|}{1-|x|}\right)$ and $n \geq 2$. Then

$$
\left(\int_{\mathbb{B}^{n}} d^{2} \phi^{2} p^{n} d x\right)\left(\int_{\mathbb{B}^{n}}|\nabla \phi|^{2} p^{n-2} d x-c 2^{n-2} \int_{\mathbb{B}^{n}} \phi^{2} d x\right) \geq\left(\frac{n-2}{2}\right)^{2}\left(\int_{\mathbb{B}^{n}} \phi^{2} p^{n} d x\right)^{2},
$$

where $c>0$. 


\section{IMPROVED WEIGHTED RELLICH-TYPE INEQUALITY}

Using the same argument as in the proof of Theorem 2.5, we prove the following improved Rellich inequality with a sharp constant.

Theorem 3.3. Let $\phi \in C_{c}^{\infty}\left(\mathbb{B}^{n} \backslash\{0\}\right), d=\log \left(\frac{1+|x|}{1-|x|}\right), n \geq 3, \alpha<2$ and $n+\alpha-4>$ 0 . Then the following inequality is valid:

$$
\int_{\mathbb{B}^{n}} d^{\alpha}\left|\Delta_{\mathbb{H}^{n}} \phi\right|^{2} d V \geq \frac{(n+\alpha-4)^{2}(n-\alpha)^{2}}{16} \int_{\mathbb{B}^{n}} d^{\alpha} \frac{\phi^{2}}{d^{4}} d V+c \epsilon 2^{n} \int_{\mathbb{B}^{n}} d^{\alpha} \phi^{2} d x
$$

where $\epsilon=\frac{(n+\alpha-4)(n-\alpha)}{8}, c>0$ and the constant $\frac{(n+\alpha-4)^{2}(n-\alpha)^{2}}{16}$ is sharp.

Proof. A straightforward computation shows that

$$
\begin{aligned}
\Delta_{\mathbb{H}}\left(d^{\alpha-2}\right) & =p^{-n} \operatorname{div}\left(p^{n-2} \nabla\left(d^{\alpha-2}\right)\right) \\
& =(\alpha-2)(\alpha-3) d^{\alpha-4}+(\alpha-2)(n-1) d^{\alpha-3}\left(\frac{p r^{2}+1}{p r}\right) .
\end{aligned}
$$

Since

$$
\frac{p r^{2}+1}{p r} \geq \frac{1}{d} \quad \text { and } \quad \alpha<2
$$

we obtain

$$
\Delta_{\mathbb{H}^{n}}\left(d^{\alpha-2}\right)=p^{-n} \operatorname{div}\left(p^{n-2} \nabla\left(d^{\alpha-2}\right)\right) \leq(\alpha-2)(n+\alpha-4) d^{\alpha-4} .
$$

Multiplying both sides of (3.13) by $\phi^{2}$ and integrating, we obtain

$$
\begin{aligned}
\int_{\mathbb{B}^{n}} \Delta_{\mathbb{H}^{n}}\left(d^{\alpha-2}\right) \phi^{2} d V & =\int_{\mathbb{B}^{n}} d^{\alpha-2} \Delta_{\mathbb{H}^{n}}\left(\phi^{2}\right) d V \\
& =2 \int_{\mathbb{B}^{n}}\left(\phi \Delta_{\mathbb{H}^{n}} \phi\right) d^{\alpha-2} d V+2 \int_{\mathbb{H}^{\prime}}\left|\nabla_{\mathbb{H}^{n}} \phi\right|^{2} d^{\alpha-2} d V \\
& \leq(\alpha-2)(n+\alpha-4) \int_{\mathbb{B}^{n}} d^{\alpha-4} \phi^{2} d V .
\end{aligned}
$$

Therefore

$$
\begin{aligned}
-2 \int_{\mathbb{B}^{n}}\left(\phi \Delta_{\mathbb{H}^{n}} \phi\right) d^{\alpha-2} d V & \geq 2 \int_{\mathbb{B}^{n}}\left|\nabla_{\mathbb{H}^{n}} \phi\right|^{2} d^{\alpha-2} d V \\
& -(\alpha-2)(n+\alpha-4) \int_{\mathbb{B}^{n}} d^{\alpha-4} \phi^{2} d V .
\end{aligned}
$$

Applying Young's inequality to the expression $-\int_{M}\left(\phi \Delta_{\mathbb{H}^{n}} \phi\right) \rho^{\alpha-2}$ in (3.14) we obtain

$$
-\int_{\mathbb{B}^{n}} d^{\alpha-2} \phi \Delta_{\mathbb{H}^{n}} \phi d V \leq \epsilon \int_{\mathbb{B}^{n}} d^{\alpha-4} \phi^{2} d V+\frac{1}{4 \epsilon} \int_{\mathbb{B}^{n}} d^{\alpha}\left|\Delta_{\mathbb{H}^{n}} \phi\right|^{2} d V,
$$

where $\epsilon>0$. Substituting (3.15) into (3.14) and using the improved Hardy inequality (3.8), we get

$\int_{\mathbb{B}^{n}} d^{\alpha}\left|\Delta_{\mathbb{H}^{n}} \phi\right|^{2} d V \geq\left(-4 \epsilon^{2}+(n+\alpha-4)(n-\alpha) \epsilon\right) \int_{\mathbb{B}^{n}} d^{\alpha-4} \phi^{2} d V+c \epsilon 2^{n} \int_{\mathbb{B}^{n}} d^{\alpha-2} \phi^{2} d x$

Observe that the quadratic function $-4 \epsilon^{2}+(n+\alpha-4)(n-\alpha) \epsilon$ attains the maximum for $\epsilon=\frac{(n+\alpha-4)(n-\alpha)}{8}$ and that this maximum is equal to $\frac{(n+\alpha-4)^{2}(n-\alpha)^{2}}{16}$. Therefore 
we have the following improved Rellich inequality:

$$
\int_{\mathbb{B}^{n}} d^{\alpha}\left|\Delta_{\mathbb{H}^{n}} \phi\right|^{2} d V \geq \frac{(n+\alpha-4)^{2}(n-\alpha)^{2}}{16} \int_{\mathbb{B}^{n}} d^{\alpha} \frac{\phi^{2}}{d^{4}} d V+c \epsilon 2^{n} \int_{\mathbb{B}^{n}} d^{\alpha-2} \phi^{2} d x .
$$

To show that the constant $\frac{(n+\alpha-4)^{2}(n-\alpha)^{2}}{16}$ is sharp, we use the following family of functions:

$$
\phi_{\epsilon}(d)= \begin{cases}\left(\frac{n+\alpha-4}{2}+\epsilon\right)(d-1)+1 & \text { if } \quad d \in[0,1], \\ d^{-\left(\frac{n+\alpha-4}{2}+\epsilon\right)} & \text { if } \quad d>1,\end{cases}
$$

and pass to the limit as $\epsilon \longrightarrow 0$.

\section{REFERENCES}

1. B. Abdellaoui, D. Colorado, I. Peral, Some improved Caffarelli-Kohn-Nirenberg inequalities, Calc. Var. Partial Differential Equations 23 (2005), no. 3, 327-345. MR2142067|(2006c:26025)

2. P. Baras and J. A. Goldstein, The heat equation with a singular potential, Trans. Amer. Math. Soc. 284 (1984), 121-139. MR742415 (85f:35099)

3. G. Barbatis, Best constants for higher-order Rellich inequalities in $L^{p}(\Omega)$, Math. Z. 255 (2007), no. 4, 877-896. MR2274540

4. G. Barbatis, S. Filippas and A. Tertikas, A unified approach to improved $L^{p}$ Hardy inequalities with best constants, Trans. Amer. Math. Soc. 356 (2004), no. 6, 2169-2196. MR2048514 (2005a:26016)

5. H. Brezis and J. L. Vázquez, Blow-up solutions of some nonlinear elliptic problems, Rev. Mat. Univ. Complutense Madrid 10 (1997), 443-469. MR.1605678 (99a:35081)

6. X. Cabré and Y. Martel, Existence versus explosion instantané pour des équations de la chaleur linéaires avec potentiel singulier, C. R. Acad. Sci. Paris Sér. I. Math. 329 (1999), 973-978. MR 1733904 (2000j:35117)

7. G. Carron, Inégalités de Hardy sur les variétés riemanniennes non-compactes, J. Math. Pures Appl. (9) 76 (1997), no. 10, 883-891. MR1489943 (99c:53026)

8. E. B. Davies and A. M. Hinz, Explicit constants for Rellich inequalities in $L_{p}(\Omega)$, Math. Z. 227 (1998), no. 3, 511-523. MR1612685 (99e:58169)

9. M. P. do Carmo and C. Xia, Complete manifolds with non-negative Ricci curvature and the Caffarelli-Kohn-Nirenberg inequalities, Compos. Math. 140 (2004), no. 3, 818-826. MR2041783 (2005b:53052)

10. L. Dupaigne, A nonlinear elliptic PDE with the inverse-square potential, J. Anal. Math. 86 (2002), 359-398. MR1894489 (2003c:35046)

11. G. B. Folland and A. Sitaram, The uncertainty principle: A mathematical survey, J. Fourier Anal. Appl. 3 (1997), 207-238. MR1448337 (98f:42006)

12. E. Fabes, C. Kenig and R. Serapioni, The local regularity of solutions of degenerate elliptic equations, Comm. Partial Differential Equations 7 (1982), 77-116. MR643158 (84i:35070)

13. J. Garcia Azorero and I. Peral, Hardy inequalities and some critical elliptic and parabolic problems, J. Diff. Equations 144 (1998), 441-476. MR1616905 (99f:35099)

14. G. Grillo, Hardy and Rellich-type inequalities for metrics defined by vector fields, Potential Analysis 18 (2003), 187-217. MR:1953228|(2003j:46043)

15. W. Heisenberg, Über den anschaulichen Inhalt der quantentheoretischen Kinematik und Mechanik, Z. Physik 43 (1927), 172-198.

16. I. Kombe, Hardy, Rellich and uncertainty principle inequalities on Carnot groups, preprint.

17. P. Li and J. Wang, Weighted Poincaré inequality and rigidity of complete manifolds, Ann. Sci. École Norm. Sup. (4) 39 (2006), no. 6, 921-982. MR2316978

18. P. Lindqvist, On the equation $\operatorname{div}\left(|\nabla u|^{p-2} \nabla u\right)+\lambda|u|^{p-2} u=0$, Proc. Amer. Math. Soc. 109 (1990), 157-164. MR 1007505 (90h:35088)

19. V. M. Miklyukov and M. L. Vuorinen, Hardy's inequality for $W_{0}^{1, p}$-functions on Riemannian manifolds, Proc. Amer. Math. Soc. 127 (1999), no. 9, 2745-2754. MR1600117 (99m:58196)

20. V. Minerbe, Weighted Sobolev inequalities and Ricci flat manifolds, preprint. 
21. I. Peral and J. L. Vázquez, On the stability or instability of the singular solution of the semilinear heat equation with exponential reaction term, Arch. Rational Mech. Anal. 129 (1995), 201-224. MR.1328476 (96b:35023)

22. F. Rellich, "Perturbation theory of eigenvalue problems", Gordon and Breach, New York, 1969. MR 0240668 (39:2014)

23. L. Sun, An Uncertainty Principle on Hyperbolic Space, Proc. Amer. Math. Soc. 121 (1994), 471-479. MR:1186137 (94h:43005)

24. J. L. Vázquez and E. Zuazua, The Hardy constant and the asymptotic behaviour of the heat equation with an inverse-square potential, J. Funct. Anal. 173 (2000), 103-153. MR.1760280 (2001j:35122)

Department of Mathematics, Dawson-Loeffler Science \& Mathematics Bldg., Oklahoma City University, 2501 N. Blackwelder, Oklahoma City, Oklahoma 73106-1493

E-mail address: ikombe@okcu.edu

Department of Mathematics, University of Oklahoma, Norman, Oklahoma 73019-0315

E-mail address: mozaydin@math.ou.edu 